INSTITUT NATIONAL DE RECHERCHE EN INFORMATIQUE ET EN AUTOMATIQUE

\title{
Feedback control for nonmonotone competition models in the chemostat
}

\author{
Gonzalo Robledo — Jean-Luc Gouzé
}

$\mathbf{N}^{\circ} \mathbf{5 0 3 3}$

3 December 2003

THÈME 4 



\title{
Feedback control for nonmonotone competition models in the chemostat
}

\author{
Gonzalo Robledo, Jean-Luc Gouzé \\ Thème 4 - Simulation et optimisation \\ de systèmes complexes \\ Projet Comore
}

Rapport de recherche $\mathrm{n}^{\circ} 5033$ - 3 December 2003 - 24 pages

\begin{abstract}
This paper deals with the problem of feedback control of competition between two species with one substrate in the chemostat with nonmonotone growth functions. Without control, the generic behavior is competitive exclusion. The aim of this paper is to find a feedback control of the dilution rate, depending only on the total biomass such that coexistence holds. We obtain a sufficient condition for the global asymptotic stability of an unique equilibrium point in the positive orthant for a three dimensional differential system which arises from this controlled competition model. This paper generalizes the results obtained by De Leenher and Smith in [7].
\end{abstract}

Key-words: Chemostat, Nonmonotone growth functions, Competition model, Feedback control, Competitive dynamical systems, Global stability. 


\section{Contrôle en boucle fermée pour des modèles de compétition non monotones dans le chemostat}

Résumé : Cet article traite le problème du contrôle en boucle fermée de la compétition entre deux espèces pour un substrat dans le chemostat avec des fonctions de croissance non monotones. Sans contrôle, le comportement asymptotique est l'exclusion compétitive. Considérant le taux de dilution comme variable de commande, l'objectif est trouver une loi de contrôle en boucle fermée dépendant uniquement de la biomasse totale qui permet la coexistence. Nous obtenons une condition suffisante pour la stabilité globale asymptotique d'un point d'équilibre unique dans l'orthant positif pour un système différentiel de dimension trois qui décrit le modèle de compétition contrôlé. Notre résultat généralise ceux obtenus dans [7].

Mots-clés : Chemostat, Fonctions de croissance non monotones, Modèle de compétition, Contrôle en boucle fermée, Systèmes dynamiques compétitifs, Stabilité globale. 


\section{Introduction}

The model presented in this paper concerns the competition and coexistence of two species in a chemostat with a single substrate. Biological motivation for chemostat models can be found in [13]. Competition theory for chemostat models predicts that the principle of competitive exclusion holds, i.e. at most one species survives and the other one tends to extinction (see [2],[13]).

In several chemostat models, control theory (see e.g. [9],[12] for a general reference) allows to obtain coexistence between species. While substrate and species are the state variables, the dilution rate and input substrate concentration can be used either or both of them as control variables. Open-loop control (e.g. periodic input) and Feedback control are two control laws that allow to reach coexistence results. In this paper we are interested in the last control law.

De Leenheer and Smith [7] studied the linear feedback control for a well known model of competition between two species and one substrate in a chemostat with monotone uptake functions, considering the dilution rate as feedback control variable and keeping the input substrate concentration at a fixed value.

However, as it has been pointed out by several works (see e.g. [3],[5],[11]), the use of monotone uptake functions cannot be valid for substrates which are growth limiting at low concentrations but are inhibitory for the species at higher concentrations. Common examples of those cases are the inhibition of Nitrobacter winogradskyi and Nitrosomas by nitrite and ammonia respectively (see [3]), the inhibition of Pseudomonas putida and Thricosporon cutaneum by phenol (see [5],[11]) and the inhibition of Candida utilis by ethanol (see [1]).

In the field of bioprocess, nonmonotone models are also widely used. The most common example is the so called Haldane model, employed in the methanogenesis step of anaerobic digestion (see e.g. [4]).

The aim of the work presented in this paper is to extend the results obtained in [7] for nonmonotone uptake functions. We have obtained sufficient conditions for the coexistence of two species; to prove our main result, we will proceed in analogy to [7]. However, nonmonotony properties of uptake functions make the study more complex than the monotone case, mainly because there are several types of nonlinearities to consider.

This paper is organized as follows: In Section 2 we have compiled some basic facts concerning to the chemostat model with nonmonotone growth functions. In Section 3 we provide an exposition of the feedback control law and show the main result of coexistence. Section 4 presents some preliminaries results related to asymptotic behavior of the model with and without competition. The proof of the main result and some extensions is stated in Section 5, robustness of the model is studied in Section 6 and a numerical example is carried out in Section 7. 


\section{Model of competition in the chemostat}

The chemostat model with competition (see [13]) is described by the differential equations:

$$
\left\{\begin{array}{l}
\dot{s}=D\left(s_{i n}-s\right)-\frac{x_{1}}{y_{1}} f_{1}(s)-\frac{x_{2}}{y_{2}} f_{2}(s), \\
\dot{x}_{1}=x_{1}\left(f_{1}(s)-D\right), \\
\dot{x}_{2}=x_{2}\left(f_{2}(s)-D\right) .
\end{array}\right.
$$

In model (1), $s$ denotes the concentration of substrate at time $t$ and $x_{i}$ denotes the biomass density of the $i$ th population of microorganisms at time $t, f_{i}(s)$ represents the per capita growth rate of nutrient of the $i$ th population and so $y_{i}$ is a growth yield constant; $D$ and $s_{i n}$ denote, respectively, the dilution rate of the chemostat and the concentration of the input substrate.

We state the general assumptions on $f_{i}(i=1,2)$ :

(F1) $f_{i}: \mathbb{R}_{+} \mapsto \mathbb{R}_{+}$and is $\mathcal{C}^{1}$.

(F2) $f_{i}(0)=0$.

(F3) $f_{i}$ is unimodal, his global maximum is noted by $s_{i}^{*}$ and has the asymptotic behavior $\lim _{t \rightarrow+\infty} f_{i}(t)=c_{i} \geq 0$.

(F4) There is $s^{*} \in\left(0, s_{i n}\right)$ such that $f_{1}\left(s^{*}\right)=f_{2}\left(s^{*}\right)=D^{*}$, moreover

$$
\left\{\begin{array}{lll}
f_{1}(s)>f_{2}(s) & \text { if } & s \in] 0, s^{*}[ \\
f_{1}(s)<f_{2}(s) & \text { if } & s \in] s^{*},+\infty[.
\end{array}\right.
$$

Assumptions (F1)-(F2) state general properties of population growth models, (F3) reflects the inhibition of growth of species $x_{1}$ and $x_{2}$ for big concentrations of substrate $s$.

An important function with properties (F1)-(F3) often found in the bioprocess research is the Haldane function:

$$
f(s)=\frac{\mu^{*} s}{K_{\mathrm{S}}+s+\frac{s^{2}}{K_{\mathrm{i}}}},
$$

where $\mu^{*}, K_{\mathrm{S}}$ and $K_{\mathrm{i}}$ are positive constants. Biological motivations for models with Haldane function can be found in [3].

Other example are the functions proposed by Sokol and Howell in [11]:

$$
f_{1}(s)=\frac{K_{1} s}{K_{2}+s^{2}}, \quad f_{2}(s)=\frac{K_{1} s}{K_{2}+s^{K_{3}}},
$$

with $K_{1}, K_{2}>0$ and $K_{3}>1$.

Assumption (F4) involves a geometrical property on the graphs of $f_{1}$ and $f_{2}$, this implies several results about asymptotic behavior of solutions of (1) as we will see later on.

INRIA 


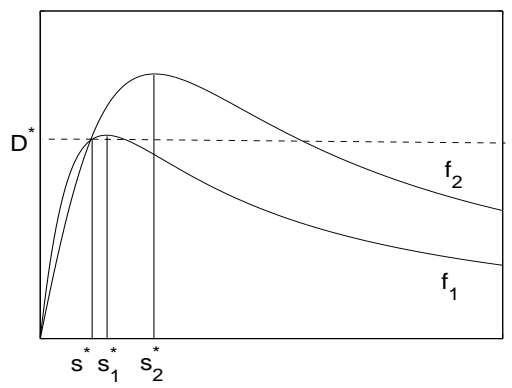

Figure 1: Case (a): $f_{2}^{\prime}\left(s^{*}\right)>f_{1}^{\prime}\left(s^{*}\right) \geq 0$, that is equivalent to $s^{*}<\min \left\{s_{1}^{*}, s_{2}^{*}\right\}$.

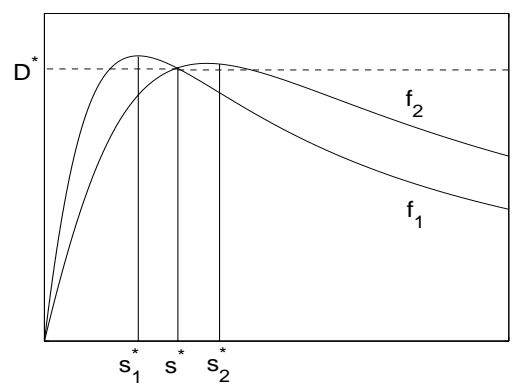

Figure 2: Case (b): $f_{1}^{\prime}\left(s^{*}\right)<0<f_{2}^{\prime}\left(s^{*}\right)$, that is equivalent to $s_{1}^{*}<s^{*}<s_{2}^{*}$.

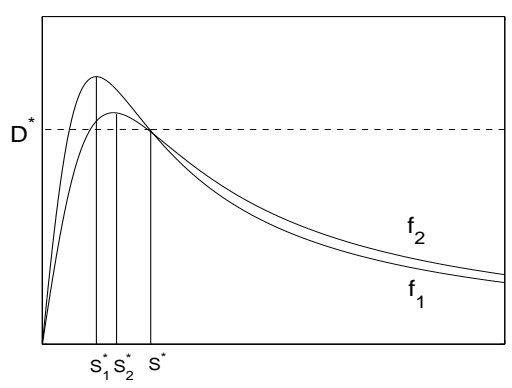

Figure 3: Case (c): $f_{1}^{\prime}\left(s^{*}\right)<f_{2}^{\prime}\left(s^{*}\right) \leq 0$, that is equivalent to $s^{*}>\max \left\{s_{1}^{*}, s_{2}^{*}\right\}$.

Remark 1 Clearly, $f_{2}^{\prime}\left(s^{*}\right)>f_{1}^{\prime}\left(s^{*}\right)$. Moreover we have three possibilities for the functions $f_{1}$ and $f_{2}$ satisfying (F1)-(F4), depending on the relative order of the intersection point $s^{*}$

$\mathrm{RR} \mathrm{n}^{\circ} 5033$ 
and the maximum points $s_{1}^{*}$ and $s_{2}^{*}$. A graphical representation of all these cases is given in Figs. 1,2 and 3 .

This model has been studied in [5] for $n$ species. Next, we consider its main result tailored for $n=2$ and functions that verify (F1)-(F4).

If $D \neq D^{*}$, there exist uniquely two defined positive real numbers $\eta_{i}$ and $\mu_{i}$ such that $\eta_{i}<\mu_{i} \leq+\infty(i=1,2)$ and

$$
\left\{\begin{array}{lll}
f_{i}(s)<D & \text { if } \quad s \notin\left[\eta_{i}, \mu_{i}\right] \\
f_{i}(s) \geq D & \text { if } \quad s \in\left[\eta_{i}, \mu_{i}\right] .
\end{array}\right.
$$

Without loss of generality we will suppose that $\max \left\{\mu_{1}, \mu_{2}\right\}<s_{\text {in }}$. Others cases can be studied similarly.

Results in [5] can be summarized coupling the relative order of numbers $\left\{s^{*}, s_{1}^{*}, s_{2}^{*}\right\}$ and $\left\{D, D^{*}\right\}$, then we have the following sub-cases corresponding to Remark (1):

\begin{tabular}{|r|c|l|}
\hline & $D<D^{*}$ & $D>D^{*}$ \\
\hline \hline$s_{1}^{*}<s^{*}<s_{2}^{*}$ & $(\mathrm{~A})$ & $(\mathrm{B})$ \\
\hline$s^{*}<\min \left\{s_{1}^{*}, s_{2}^{*}\right\}$ & $(\mathrm{C})$ & $(\mathrm{D})$ \\
\hline$s^{*}>\max \left\{s_{1}^{*}, s_{2}^{*}\right\}$ & $(\mathrm{E})$ & $(\mathrm{F})$ \\
\hline
\end{tabular}

Proposition 1 [5, Butler and Wolkowicz] With the exception of a set of initial conditions of Lebesgue measure zero, all solutions of (1) are initial condition dependent and satisfy:

- Cases (A),(C),(E) and (F):

$\lim _{t \rightarrow+\infty}\left(s(t), x_{1}(t), x_{2}(t)\right)=\left(\eta_{1}, y_{1}\left[s_{i n}-\eta_{1}\right], 0\right)$ or

$\lim _{t \rightarrow+\infty}\left(s(t), x_{1}(t), x_{2}(t)\right)=\left(s_{i n}, 0,0\right)$.

- Case (B):

$\lim _{t \rightarrow+\infty}\left(s(t), x_{1}(t), x_{2}(t)\right)=\left(\eta_{1}, y_{1}\left[s_{i n}-\eta_{1}\right], 0\right)$,

$\lim _{t \rightarrow+\infty}\left(s(t), x_{1}(t), x_{2}(t)\right)=\left(\eta_{2}, 0, y_{2}\left[s_{i n}-\eta_{2}\right]\right)$ or

$\lim _{t \rightarrow+\infty}\left(s(t), x_{1}(t), x_{2}(t)\right)=\left(s_{i n}, 0,0\right)$.

- Case (D):

$\lim _{t \rightarrow+\infty}\left(s(t), x_{1}(t), x_{2}(t)\right)=\left(\eta_{2}, 0, y_{2}\left[s_{i n}-\eta_{2}\right]\right)$ or

$\lim _{t \rightarrow+\infty}\left(s(t), x_{1}(t), x_{2}(t)\right)=\left(s_{i n}, 0,0\right)$.

Note that Proposition 1 is a result qualitatively different from the model with functions $f_{i}$ strictly increasing: the novelty is that extinction of two species can be expected because $\left(s_{i n}, 0,0\right)$ is a locally asymptotically stable solution (see e.g. [5],[13] for details).

INRIA 


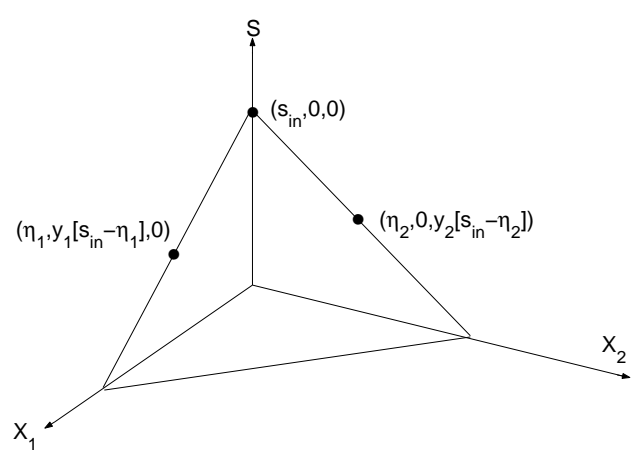

Figure 4: Attractor of system (1)

In Figure 4, we have sketched the attractor of system (1) which is composed by three critical points.

The special case $D=D^{*}$ is not considered in [5], there is a set of nonhyperbolic critical points of equation (1) in the straight line:

$$
\Sigma=\left\{\left(x_{1}, x_{2}\right) \in \mathbb{R}_{+}^{2}: \frac{x_{1}}{y_{1}}+\frac{x_{2}}{y_{2}}=s_{i n}-s^{*}\right\} .
$$

In the remainder of this paper we assume that $y_{1} \neq y_{2}$. In the sequel $y_{\min }, y_{\max }$ denote $\min \left\{y_{1}, y_{2}\right\}$ and $\max \left\{y_{1}, y_{2}\right\}$ respectively.

\section{The uniform persistence in a control setting}

Until now, we have used the term coexistence as the survival of the two species. Henceforth, we will use the concepts of persistence and uniform persistence. We recall the definitions given by Butler et al in [6]:

Definition $1 A$ component $x_{i}(t)$ of a given $O D E$ system is said to be persistent if for any $x_{i}(0)>0$ it follows that $x_{i}(t)>0$ for all $t>0$ and $\liminf _{t \rightarrow+\infty} x_{i}(t)>0$.

If there exists $\delta>0$ independent of $x_{i}(0)$ such that component $x_{i}(t)$ is persistent and $\liminf _{t \rightarrow+\infty} x_{i}(t)>\delta$, then $x_{i}(t)$ is uniformly persistent.

Uniform persistence of the species is usually observed as the existence of a globally attracting periodic solution or a globally asymptotically stable solution. As we have seen in Proposition 1, persistence of two species is not possible in system (1).

$\mathrm{RR} \mathrm{n}^{\circ} 5033$ 


\subsection{The feedback control problem}

In several works (see e.g. [13]), uniform persistence of competition models in chemostat has been obtained considering the input $s_{i n}$ or the dilution rate $D$ as periodic functions. In this paper we will follow another approach, using control theory and feedback control with dilution rate $D$. Our goal is to obtain sufficient conditions for uniform persistence considering the following hypotheses:

Hypothesis 1 (Control Hypothesis) Dilution rate D is the feedback control variable.

Hypothesis 2 (Output Hypothesis) The only output available is:

$$
y=x_{1}+x_{2} .
$$

Output hypothesis is considered because in several cases, technical difficulties do not allow to measure $x_{1}$ and $x_{2}$ independently and it is necessary to consider total biomass. For example, the measurement is done often by photometric methods (see [11] and the references given there) that do not allow to distinguish between the two species.

We define the feedback control law $D: \mathbb{R}_{+}^{2} \mapsto \mathbb{R}_{+}$by:

$$
D\left(x_{1}, x_{2}\right)=g\left(x_{1}+x_{2}\right) .
$$

We also make the following assumptions on the function $g$ :

(G1) $g: \mathbb{R}_{+} \mapsto \mathbb{R}_{+}$is $\mathcal{C}^{1}$ and Lipschitz.

(G2) $g(0) \in\left[0, f_{1}\left(s_{i n}\right)\right), g$ is strictly increasing and there is $s_{c}>0$ such that $g\left(s_{c}\right)=D^{*}$.

Replacing $D$ by the feedback control law (3), system (1) becomes:

$$
\left\{\begin{array}{l}
\dot{s}=g\left(x_{1}+x_{2}\right)\left(s_{i n}-s\right)-\frac{x_{1}}{y_{1}} f_{1}(s)-\frac{x_{2}}{y_{2}} f_{2}(s), \\
\dot{x}_{1}=x_{1}\left(f_{1}(s)-g\left(x_{1}+x_{2}\right)\right) \\
\dot{x}_{2}=x_{2}\left(f_{2}(s)-g\left(x_{1}+x_{2}\right)\right)
\end{array}\right.
$$

Remark 2 Non negativity of function $g$ is supposed because dilution rate $D$ cannot be negative. Assumption (G1) ensures existence and uniqueness of the initial value problem and (G2) implies existence of a new critical point.

\subsection{Choice of the control}

Our goal is to obtain sufficient conditions on the function $g$ and its relations with $f_{1}$ and $f_{2}$ to have existence and global asymptotic stability of the interior critical point.

First, let us define the following equations that will be used for to study the asymptotic behavior of system (4):

$$
\begin{aligned}
& f_{1}(s)-g\left(y_{1}\left[s_{i n}-s\right]\right)=0 . \\
& f_{2}(s)-g\left(y_{2}\left[s_{i n}-s\right]\right)=0 .
\end{aligned}
$$

We will make the assumptions 


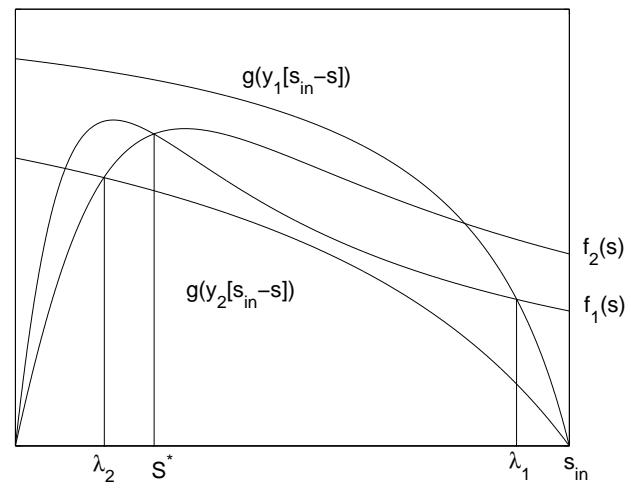

Figure 5: Geometrical interpretation of (H1)-(H2).

(H1) The following inequality holds:

$$
g\left(y_{\max }\left[s_{i n}-s^{*}\right]\right)>D^{*}>g\left(y_{\min }\left[s_{i n}-s^{*}\right]\right) .
$$

(H2) Eqs. (5) and (6) have one positive solution $\lambda_{1}$ and $\lambda_{2}$ respectively. Moreover, if $y_{1}>y_{2}$ we have that $\lambda_{1} \in\left(s^{*}, s_{i n}\right)$ and $\lambda_{2} \in\left(0, s^{*}\right)$.

(H3) The following inequality holds:

$$
y_{\min } g^{\prime}\left(x_{1}+x_{2}\right)>-f_{1}^{\prime}\left(s_{i n}-\frac{x_{1}}{y_{1}}-\frac{x_{2}}{y_{2}}\right) \quad \text { for all } \quad\left(x_{1}, x_{2}\right) \in \mathcal{O} .
$$

(H4) The following inequality holds:

$$
y_{\min } g^{\prime}\left(x_{1}+x_{2}\right)>-f_{2}^{\prime}\left(s_{i n}-\frac{x_{1}}{y_{1}}-\frac{x_{2}}{y_{2}}\right) \quad \text { for all } \quad\left(x_{1}, x_{2}\right) \in \mathcal{O} .
$$

Where

$$
\mathcal{O}=\left\{\left(x_{1}, x_{2}\right) \in \mathbb{R}_{+}^{2}: 0 \leq \frac{x_{1}}{y_{1}}+\frac{x_{2}}{y_{2}} \leq s_{i n}\right\}
$$

Remark 3 As we can choose a strictly increasing function g, assumptions (H1)-(H2) are always satisfied with reasonable choices. In fact, those assumptions can be interpreted geometrically with the graph of functions defined in equations (5) and (6) (see Fig. 5).

Note that, in some cases can be rather difficult to find a function $g$ checking assumptions (H3)-(H4). Otherwise, if $s_{1}^{*} \geq s_{i n}$ (respectively $s_{2}^{*} \geq s_{i n}$ ) then assumption (H3) (respectively $(\mathbf{H 4}))$ is always verified. 
Inequality $y_{1} \neq y_{2}$ implies that system (4) has a critical point $\left(s^{*}, x_{1}^{*}, x_{2}^{*}\right)$ defined by

$$
x_{1}^{*}=\frac{y_{1}\left[y_{2}\left(s_{i n}-s^{*}\right)-g^{-1}\left(D^{*}\right)\right]}{y_{2}-y_{1}}, \quad x_{2}^{*}=\frac{y_{2}\left[g^{-1}\left(D^{*}\right)-y_{1}\left(s_{i n}-s^{*}\right)\right]}{y_{2}-y_{1}} .
$$

Assumption (H1) implies that $\left(s^{*}, x_{1}^{*}, x_{2}^{*}\right) \in \operatorname{int} \mathbb{R}_{+}^{3}$, (H2) implies that there are two hyperbolic critical points of system (4) in the boundary of $\mathbb{R}_{+}^{3}$ defined by:

$$
E_{1}=\left(\lambda_{1}, y_{1}\left[s_{i n}-\lambda_{1}\right], 0\right) \text { and } E_{2}=\left(\lambda_{2}, 0, y_{2}\left[s_{i n}-\lambda_{2}\right]\right) .
$$

Finally, note that if $g(0)=0$, then

$$
\Lambda=\left\{\left(s, x_{1}, x_{2}\right) \in \mathbb{R}_{+}^{3}: \quad s \geq 0, \quad x_{1}=x_{2}=0\right\}
$$

is a set of nonhyperbolic critical points of the system (4).

In the remainder of this paper we assume that the initial conditions of system (4) are in $\mathbb{R}_{+}^{3} \backslash \Lambda$.

\subsection{Main Result}

The main result of this paper provides a sufficient condition for the global asymptotic stability of the critical point $\left(s^{*}, x_{1}^{*}, x_{2}^{*}\right)$.

Theorem 1 Let $y_{\max }=y_{1}$, if at least one of the following conditions is verified:

(i) Assumptions (H1)-(H4) holds

(ii) Assumptions (H1)-(H3) holds and inequalities $s^{*}<\min \left\{s_{1}^{*}, s_{2}^{*}\right\}$ or $s^{*} \in\left(s_{1}^{*}, s_{2}^{*}\right)$ are verified

(iii) Assumptions (H1)-(H2) holds and $s^{*}<\min \left\{s_{1}^{*}, s_{2}^{*}\right\}$

then, the critical point $\left(s^{*}, x_{1}^{*}, x_{2}^{*}\right)$ is a globally asymptotically stable solution of system (4) for all initial condition in int $\mathbb{R}_{+}^{3}$.

Note that the relative order of points $s^{*}, s_{1}^{*}$ and $s_{2}^{*}$ summarized in Remark 1 implies different requirements on assumptions (H1)-(H4). This is important, because assumption (H4) is unnecessarily restrictive for the case (ii) and assumptions (H3)-(H4) are unnecessarily restrictive for the case (iii). Furthermore, as we have pointed out in Remark 3, there are some cases where checking assumptions (H3)-(H4) can be rather complicated.

\section{Preliminary results}

In the following results, we establish some properties related to the asymptotic behavior of solutions which are needed in the proof of Theorem 1.

INRIA 
Lemma 1 Let $\left(s(t), x_{1}(t), x_{2}(t)\right)$ be a solution of system (4) with initial condition in int $\mathbb{R}_{+}^{3}$. Then this solution is bounded and verifies:

$$
\lim _{t \rightarrow+\infty} s+\frac{x_{1}}{y_{1}}+\frac{x_{2}}{y_{2}}=s_{i n} .
$$

Proof: The main idea of the proof is taken of [10]. Let $V: \mathbb{R}_{+}^{3} \mapsto \mathbb{R}_{+}$defined by:

$$
V\left(s, x_{1}, x_{2}\right)=\left(s+\frac{x_{1}}{y_{1}}+\frac{x_{2}}{y_{2}}-s_{i n}\right) .
$$

Clearly, $V^{\prime}=-D\left(x_{1}, x_{2}\right) V$. If $g(0)>0$ the result is a consequence of LaSalle invariance principle. If $g(0)=0$ we have that $D\left(x_{1}, x_{2}\right) \geq 0$ and it is easy to check that every solution of system (4) is bounded and uniformly continuous on $[0, \infty)$.

Clearly, Eq. (9) holds if and only if:

$$
\lim _{t \rightarrow+\infty} \int_{0}^{t} D\left(x_{1}(s), x_{2}(s)\right) d s=+\infty .
$$

Conversely, if we suppose that

$$
\lim _{t \rightarrow+\infty} \int_{0}^{t} D\left(x_{1}(s), x_{2}(s)\right) d s<+\infty
$$

it is easily seen that the function $t \mapsto D\left(x_{1}(t), x_{2}(t)\right)$ is uniformly continuous, therefore Barbălat's lemma (see e.g. [9]) yields

$$
\lim _{t \rightarrow+\infty} g\left(x_{1}(t)+x_{2}(t)\right)=0 .
$$

By continuity of $g$ we have that

$$
\lim _{t \rightarrow+\infty} \frac{\exp \left[\int_{0}^{t} f_{i}(s(u)) d u\right]}{\exp \left[\int_{0}^{t} g\left(x_{1}(u)+x_{2}(u)\right) d u\right]}=\lim _{t \rightarrow+\infty} x_{i}(t)=0
$$

and it follows that

$$
\lim _{t \rightarrow+\infty} \exp \left(\int_{0}^{t} f_{i}(s(u)) d u\right)=0
$$

but this is not possible, hence (9) holds, which completes the proof.

If $g(0)=0$, it follows by Lemma 1 that critical points in $\Lambda \backslash\left\{\left(s_{i n}, 0,0\right)\right\}$ are not attractive.

We will denote by $U_{1}$ and $U_{2}$ the positively invariant sets:

$$
\begin{aligned}
& U_{1}=\left\{\left(s, x_{1}, x_{2}\right) \in \mathbb{R}_{+}^{3}: s \geq 0, x_{1}>0 \quad \text { and } \quad x_{2}=0\right\} \\
& U_{2}=\left\{\left(s, x_{1}, x_{2}\right) \in \mathbb{R}_{+}^{3}: s \geq 0, x_{2}>0 \quad \text { and } \quad x_{1}=0\right\} .
\end{aligned}
$$

$\mathrm{RR} \mathrm{n}^{\circ} 5033$ 
As we are interested in persistence of species $x_{1}$ and $x_{2}$, it is important to know if each species is persistent in the chemostat without competition. Each species must be able to survive alone in the chemostat if it is to be able to survive with a competitor. The following result gives an affirmative answer.

Lemma 2 Let $\left(s(t), x_{1}(t), x_{2}(t)\right)$ be a solution of system (4) with initial condition in $U_{i}$ $(i=1,2)$. Then this solution is bounded and verifies:

$$
\begin{gathered}
\lim _{t \rightarrow+\infty} s+\frac{x_{i}}{y_{i}}=s_{i n} . \\
\lim _{t \rightarrow+\infty} x_{i}(t)=y_{i}\left[s_{i n}-\lambda_{i}\right] \text { and } \lim _{t \rightarrow+\infty} s(t)=\lambda_{i} .
\end{gathered}
$$

Proof: We give the proof for the case $i=1$; the other case is similar. Eq. (10) is an immediate consequence of Lemma 1 . Clearly, $x_{2}(t)=0$ for $t \geq 0$. We consider the second equation of system (4) and insert the solution $s(t)$ initiated at $s(0)$. Then we obtain the following nonautonomous differential equation:

$$
\dot{x}_{1}=x_{1}\left(f_{1}(s(t))-g\left(x_{1}\right)\right) .
$$

By (10), it follows that for each initial condition $s(0)$, Eq. (12) is asymptotically autonomous (see e.g. [16] for details) with limit equation:

$$
\dot{z}_{1}=z_{1}\left(f_{1}\left(s_{i n}-\frac{z_{1}}{y_{1}}\right)-g\left(z_{1}\right)\right) .
$$

Assumption (H2) implies that the solution $z_{1}(t)$ of the Eq. (13) satisfies

$$
\lim _{t \rightarrow+\infty} z_{1}(t)=y_{1}\left[s_{\text {in }}-\lambda_{1}\right] .
$$

Applying corollary 4.3 from [16], it follows that solutions of (12) converge to $y_{1}\left[s_{i n}-\lambda_{1}\right]$ and Eq. (10) makes it obvious that $\lim _{t \rightarrow+\infty} s(t)=s^{*}$, which proves the Lemma.

Lemma 3 If $y_{\max }=y_{1}$, then every component $x_{i}(t)$ of a solution of system (4) with initial condition in int $\mathbb{R}_{+}^{3}$ is uniformly persistent.

Proof: Let $\mathrm{X}=\left\{\left(s, x_{1}, x_{2}\right) \in \mathbb{R}_{+}^{3}: s \leq s_{i n}, x_{1}+x_{2} \leq L\right\}$, where $L>y_{1} s_{i n}$ and $g(L)>\max \left\{f_{1}\left(s_{1}^{*}\right), f_{2}\left(s_{2}^{*}\right)\right\}$. Lemma 1 implies that $\mathrm{X}$ is positively invariant and every solution of system (4) reach $\mathrm{X}$ in finite time and cannot leave it. Hence, we can consider only initial conditions in $\mathrm{X}$.

Let $M=\mathrm{X} \cap\left(U_{1} \cup U_{2}\right)$, following the paper of Hofbauer [8], we will prove that $M$ is a repeller set, that is equivalent to uniform persistence. Next, we build the average Lyapunov function $P: \mathrm{X} \mapsto \mathbb{R}$, defined by

$$
P\left(x_{1}, x_{2}\right)=x_{1} x_{2}
$$

INRIA 
Clearly, $P\left(x_{1}, x_{2}\right)=0$ for $\left(x_{1}, x_{2}\right) \in M$ and $P\left(x_{1}, x_{2}\right)>0$ for $\left(x_{1}, x_{2}\right) \in \mathrm{X} \backslash M$. Moreover, $\dot{P}\left(x_{1}, x_{2}\right)=\Psi\left(s, x_{1}, x_{2}\right) P\left(x_{1}, x_{2}\right)$ where $\Psi: \mathrm{X} \mapsto \mathbb{R}$ is the continuous function:

$$
\Psi\left(s, x_{1}, x_{2}\right)=f_{1}(s)+f_{2}(s)-2 g\left(x_{1}+x_{2}\right) .
$$

Let $W^{s}\left(E_{i}\right)$ and $W^{u}\left(E_{i}\right)$ be the stable and unstable manifold respectively of critical points $E_{i}$. By $y_{\max }=y_{1}$ and Lemma 2 , we have that $U_{i}$ are included in $W^{s}\left(E_{i}\right)$; moreover, (H2) implies that $E_{i}$ are saddle-points and $\Psi\left(E_{i}\right)>0$.

Finally, continuity of $\Psi$ implies that for all initial condition $\vec{z}(0) \in M$ there is a time $T>0$ such that

$$
\int_{0}^{T} \Psi(\vec{z}(t)) d t>0 .
$$

Applying corollaries 1 and 2 from [8], it follows that $M$ is a repeller set and the proof is complete.

\section{Proof of main result}

Let us return to system (4) into the equations $\dot{x}_{1}$ and $\dot{x}_{2}$ and insert the solution $s(t)$ initiated at $s(0)$. Then, for each initial condition $s(0)$ we obtain the nonautonomous system:

$$
\left\{\begin{array}{l}
\dot{x}_{1}=x_{1}\left(f_{1}(s(t))-g\left(x_{1}+x_{2}\right)\right), \\
\dot{x}_{2}=x_{2}\left(f_{2}(s(t))-g\left(x_{1}+x_{2}\right)\right) .
\end{array}\right.
$$

Note that Lemma 1 implies that for each initial condition $s(0)$, the system (14) is asymptotically autonomous with limit system:

$$
\left\{\begin{array}{l}
\dot{z}_{1}=z_{1}\left(f_{1}\left(s_{i n}-\frac{z_{1}}{y_{1}}-\frac{z_{2}}{y_{2}}\right)-g\left(z_{1}+z_{2}\right)\right), \\
\dot{z}_{2}=z_{2}\left(f_{2}\left(s_{i n}-\frac{z_{1}}{y_{1}}-\frac{z_{2}}{y_{2}}\right)-g\left(z_{1}+z_{2}\right)\right) .
\end{array}\right.
$$

Moreover, system (15) defines a dynamical system in the set $\mathcal{O} \subset \mathbb{R}_{+}^{2}$ and the relation between asymptotic behavior of both systems is summarized by the following result:

Proposition 2 [16, Thieme] Let $\omega$ be the $\omega$-limit of a forward bounded solution of (14). Assume that there exists a neighborhood of $\omega$ which contains at most finitely many equilibria of (15). Then the following trichotomy holds :

(a) $\omega$ consists of an equilibrium of (15).

(b) $\omega$ is the union of periodic orbits of (15) and possibly of centers of (15) that are surrounded by periodic orbits living in $\omega$.

(c) $\omega$ contains equilibria of (15) that are cyclically chained to each other in $\omega$ by orbits of (15).

$\mathrm{RR} \mathrm{n}^{\circ} 5033$ 
The critical points of system (15) are the projections in the set $\mathcal{O}$ of the hyperbolic critical points stated in previous section beside $\left(s_{i n}, 0,0\right) \in \Lambda$.

$$
\begin{aligned}
& E_{0}^{p}=(0,0) \\
& E_{1}^{p}=\left(y_{1}\left[s_{i n}-\lambda_{1}\right], 0\right) \\
& E_{2}^{p}=\left(0, y_{2}\left[s_{i n}-\lambda_{2}\right]\right) \\
& E_{s}^{p}=\left(x_{1}^{*}, x_{2}^{*}\right) .
\end{aligned}
$$

The local properties of critical points of (15) are summarized in the following Lemma:

Lemma 4 Let assumptions $(\mathbf{H 1})-(\mathbf{H 2})$ and $y_{\max }=y_{1}$ hold. Then all the critical points of (15) are hyperbolic, moreover:

(a) Critical point $E_{0}^{p}$ is a repeller.

(b) Critical points $E_{i}^{p}$ are saddle-points, $W^{u}\left(E_{i}^{p}\right)$ are in $\operatorname{int} \mathbb{R}_{+}^{2}(i=1,2)$ and

$$
\begin{aligned}
& W^{s}\left(E_{1}^{p}\right)=\left\{\left(z_{1}, z_{2}\right) \in \mathcal{O} \mid 0<z_{1}<y_{1} s_{i n} \quad \text { and } \quad z_{2}=0\right\}, \\
& W^{s}\left(E_{2}^{p}\right)=\left\{\left(z_{1}, z_{2}\right) \in \mathcal{O} \mid 0<z_{2}<y_{2} s_{i n} \quad \text { and } \quad z_{1}=0\right\} .
\end{aligned}
$$

Moreover, $E_{1}^{p}$ and $E_{2}^{p}$ cannot belong to $\omega(\vec{z}(0))$ when $\vec{z}(0) \in \operatorname{int} \mathcal{O}$.

(c) Local asymptotic stability of critical point $E_{s}^{p}$ is always verified when $s^{*}<\min \left\{s_{1}^{*}, s_{2}^{*}\right\}$, is verified by assumption $(\mathbf{H 3})$ when $s^{*} \in\left(s_{1}^{*}, s_{2}^{*}\right)$ and by assumptions $(\mathbf{H 3})$ and $(\mathbf{H 4})$ when $s^{*}>\max \left\{s_{1}^{*}, s_{2}^{*}\right\}$.

Proof: Result (a) is obtained from the standard linearization procedure and (G2). Result (b) is obtained following the lines of the proof of Lemma 3. Finally, as $y_{\max }=y_{1}$ a necessary and sufficient condition for local stability of $E_{s}^{p}$ is

$$
1>-\frac{\frac{x_{1}^{*}}{y_{1}} f_{1}^{\prime}\left(s^{*}\right)+\frac{x_{2}^{*}}{y_{2}} f_{2}^{\prime}\left(s^{*}\right)}{\left(x_{1}^{*}+x_{2}^{*}\right) g^{\prime}\left(x_{1}^{*}+x_{2}^{*}\right)} .
$$

Now, the proof of result (c) is straightforward.

The proof of the Theorem will be divided into 3 steps:

1. Let $\vec{x}(0) \in \operatorname{int} \mathbb{R}_{+}^{2}$ an initial condition of system (14). We will proof that system (15) cannot have periodic orbits or a cycle of critical points. An important consequence of Proposition 2 is that the set $\omega(\vec{x}(0))$ is a critical point of system (15).

2. Lemma 3 implies that this critical point cannot be in $\partial \mathcal{O}$, hence $\omega(\vec{x}(0))=\left(x_{1}^{*}, x_{2}^{*}\right)$.

3. Finally, Eq. (9) makes it obvious that $\lim _{t \rightarrow+\infty} s(t)=s^{*}$, which proves the Theorem. 


\subsection{Proof of case (i)}

Let $\vec{z}(0) \in \operatorname{int} \mathcal{O}$ be an initial condition of system (15). The asymptotic behavior of a solution with this initial condition is described by the following Lemma:

Lemma 5 Let $\vec{z}(t)$ be a solution of system (15) with initial condition $\vec{z}(0)$, then there exist a real number $T>0$ such that the solutions $z_{i}(t)$ are monotone on $t>T$. In particular we know that $\omega(\vec{z}(0))$ is a critical point.

Proof: By assumptions (H3)-(H4) we have that system (15) is competitive on $\mathcal{O}$ (i.e. the off-diagonal entries of the Jacobian matrix on $\mathcal{O}$ are negative or zero). As the forward orbit of $\vec{z}(0)$ is a relatively compact set, we apply Theorem 3.2.2 from [15] and the Lemma follows.

A consequence is that system (15) cannot have periodic orbits or a cycle of critical points, which proves the Theorem.

Assume now that $s^{*}<\min \left\{s_{1}^{*}, s_{2}^{*}\right\}$ or $s^{*} \in\left(s_{1}^{*}, s_{2}^{*}\right)$ and $(\mathbf{H} 4)$ is not verified. Note that in this case, system (15) is not necessary competitive and Lemma 5 cannot be applied.

As before, let $\vec{z}(t)$ be a solution of system (15) with initial condition $\vec{z}(0) \in \operatorname{int} \mathcal{O}$. We will prove that $\vec{z}(t)$ cannot be a periodic orbit and that $\omega(\vec{z}(0))$ cannot be a cycle of critical points.

\subsection{Proof of case (ii)}

Let $\hat{s} \in\left(s^{*}, s_{2}^{*}\right)$. We define an increasing $\mathcal{C}^{1}$-function $e_{2}:[\hat{s},+\infty) \mapsto \mathbb{R}$ such that $e_{2}^{(k)}(\hat{s})=f_{2}^{(k)}(\hat{s})$ for $k=0,1$. Let us denote by $m_{2}$ the increasing envelope of $f_{2}$ as the function:

$$
m_{2}(s)=\left\{\begin{array}{lll}
f_{2}(s) & \text { if } \quad s \in[0, \hat{s}] \\
e_{2}(s) & \text { if } \quad s \geq \hat{s} .
\end{array}\right.
$$

Let us consider the system:

$$
\left\{\begin{array}{l}
\dot{u}_{1}=u_{1}\left(f_{1}\left(s_{i n}-\frac{u_{1}}{y_{1}}-\frac{u_{2}}{y_{2}}\right)-g\left(u_{1}+u_{2}\right)\right), \\
\dot{u}_{2}=u_{2}\left(m_{2}\left(s_{i n}-\frac{u_{1}}{y_{1}}-\frac{u_{2}}{y_{2}}\right)-g\left(u_{1}+u_{2}\right)\right), \\
u_{1}(0)=z_{1}(0)>0, u_{2}(0)=z_{2}(0)>0 .
\end{array}\right.
$$

Note that system (18) has the same critical points as system (15) with the same local properties summarized by Lemma 4. Assumption (H3) implies that the system (18) is competitive and replacing $f_{2}$ by $m_{2}$ in the case (i) of Theorem 1 we have that:

$$
\lim _{t \rightarrow+\infty}\left(u_{1}(t), u_{2}(t)\right)=\left(x_{1}^{*}, x_{2}^{*}\right) .
$$

Using the order $K_{(0,1)}$ and Proposition 3 (see Appendix) we have the inequalities:

$$
\left(z_{1}(0), z_{2}(0)\right) \geq_{K_{(0,1)}}\left(u_{1}(0), u_{2}(0)\right) \geq_{K_{(0,1)}}\left(0, u_{2}(0)\right)
$$


and

$$
\left(z_{1}(t), z_{2}(t)\right) \geq_{K_{(0,1)}}\left(u_{1}(t), u_{2}(t)\right) \geq_{K_{(0,1)}}\left(0, u_{2}(t)\right)
$$

for all $t \geq 0$. Letting $t \rightarrow+\infty$, we have that:

$$
\liminf _{t \rightarrow+\infty} z_{1}(t) \geq x_{1}^{*} \quad \text { and } \quad \limsup _{t \rightarrow+\infty} z_{2}(t) \leq x_{2}^{*} \leq y_{2}\left[s_{i n}-\lambda_{2}\right] .
$$

This gives that $\omega(\vec{z}(0))$ is a subset of $\left\{\left(x_{1}, x_{2}\right) \in \mathcal{O} \mid x_{1} \geq x_{1}^{*} \quad, 0<x_{2} \leq x_{2}^{*}\right\}$, hence $\vec{z}(t)$ cannot be a periodic orbit.

It remains to prove that there is not a cycle of critical points. If we suppose the existence of one, Lemma 4 implies that $E_{0}^{p}$ is a repeller and $E_{s}^{p}$ is locally asymptotically stable, hence they cannot belong to this cycle. Moreover, Eq. (19) implies that $E_{2}^{p}$ cannot belong to this cycle, so only $E_{1}^{p}$ could possibly belong to it.

But Lemma 4 implies that $W^{s}\left(E_{1}^{p}\right) \cap W^{u}\left(E_{1}^{p}\right) \backslash E_{1}^{p}=\emptyset$, hence $E_{1}^{p}$ cannot belong to this cycle, which proves the Theorem.

\subsection{Proof of case (iii)}

Let $\hat{s} \in\left(s^{*}, \max \left\{s_{1}^{*}, s_{2}^{*}\right\}\right)$. We define a couple of continuous increasing functions $e_{1}, e_{2}$ : $[\hat{s},+\infty) \mapsto \mathbb{R}$ such that $e_{i}^{(k)}(\hat{s})=f_{i}^{(k)}(\hat{s})$ for $k=0,1$ and $e_{2}(s)>e_{1}(s)$ for all $s>\hat{s}$. Let us denote by $m_{i}$ the increasing envelope of $f_{i}$ as the functions:

$$
m_{i}(s)=\left\{\begin{array}{lll}
f_{i}(s) & \text { if } & s \in[0, \hat{s}] \\
e_{i}(s) & \text { if } \quad s \geq \hat{s}
\end{array}\right.
$$

Let us consider the system:

$$
\left\{\begin{array}{l}
\dot{u}_{1}=u_{1}\left(m_{1}\left(s_{i n}-\frac{u_{1}}{y_{1}}-\frac{u_{2}}{y_{2}}\right)-g\left(u_{1}+u_{2}\right)\right), \\
\dot{u}_{2}=u_{2}\left(m_{2}\left(s_{i n}-\frac{u_{1}}{y_{1}}-\frac{u_{2}}{y_{2}}\right)-g\left(u_{1}+u_{2}\right)\right), \\
u_{1}(0)>0, u_{2}(0)>0 .
\end{array}\right.
$$

Note that system (21) is competitive and has the same interior critical point as (15). If we suppose that there is a solution of system (15) such that is a periodic orbit parametrized by $\vec{\psi}(t)$. We can consider the backward orbits of systems (15) and (21), note that this orbit is a solution of reversed time cooperative system:

$$
\left\{\begin{array}{l}
\dot{v}_{1}=-v_{1}\left(m_{1}\left(s_{i n}-\frac{v_{1}}{y_{1}}-\frac{v_{2}}{y_{2}}\right)-\alpha g\left(v_{1}+v_{2}\right)\right), \\
\dot{v}_{2}=-v_{2}\left(m_{2}\left(s_{i n}-\frac{v_{1}}{y_{1}}-\frac{v_{2}}{y_{2}}\right)-\alpha g\left(v_{1}+v_{2}\right)\right), \\
v_{1}(0)=u_{1}(0)>0, v_{2}(0)=u_{2}(0)>0
\end{array}\right.
$$

We choose the initial conditions of systems such that

$$
z_{1}(0)=\psi_{1}(0)>v_{1}(0)=x_{1}^{*}, \quad z_{2}(0)=\psi_{2}(0)>v_{2}(0)=x_{2}^{*} .
$$


Applying theorem B.1 from [13], it follows that

$$
\psi_{1}(t)>x_{1}^{*} \quad \text { and } \quad \psi_{2}(t)>x_{2}^{*} \text { for all } t<0
$$

and we obtain a contradiction.

It remains to prove that there is not a cycle of critical points. If we suppose the existence of one, as in the proof of case (ii) Lemma 4 implies that $E_{0}^{p}$ and $E_{s}^{p}$ cannot belong to this cycle, so only $E_{1}^{s}$ and/or $E_{2}^{s}$ could possibly belong to it.

By Lemma 4 we have that $W^{u}\left(E_{1}^{p}\right) \cap W^{s}\left(E_{2}^{p}\right)=\emptyset$ and $W^{u}\left(E_{2}^{p}\right) \cap W^{s}\left(E_{1}^{p}\right)=\emptyset$, then there is not a cycle connecting $E_{1}^{p}$ and $E_{2}^{p}$.

Finally, as in the proof of case (ii), is not possible the existence of a cycle connecting $E_{i}^{p}$ $(i=1,2)$ to itself, which proves the Theorem.

\section{Robustness of model}

We consider the case when the uptake functions $f_{i}$ of system (4) are, in some sense, unknown. Usually, the formulation of uptake functions is based on experimental evidence with measurement error (see e.g. [11]). Thus, we are not able to obtain a analytic form of the functions, but only some qualitative properties and quantitative bounds. Our goal is to obtain sufficient conditions for the uniform persistence in such cases.

We will suppose that the following properties are satisfied:

(R1) $f_{1}$ and $f_{2}$ are functionally bounded, i.e. there exist a couple of well known maps $l_{i}$ and $u_{i}$, such that satisfy assumptions (F1)-(F4) (with maximums noted by $s_{i-}^{*}$ and $s_{i+}^{*}$ respectively) and verify

$$
l_{i}(s) \leq f_{i}(s) \leq u_{i}(s), \quad s \geq 0, \quad i=1,2 .
$$

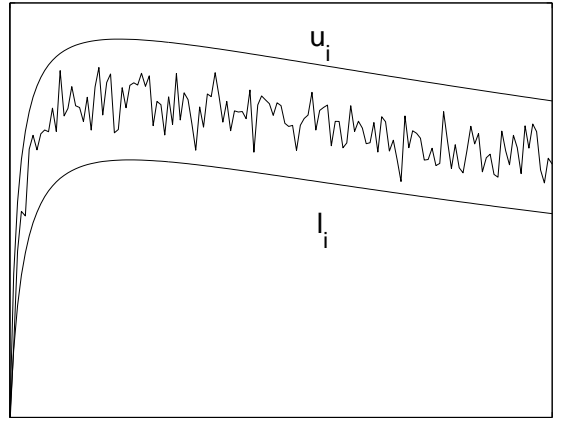

Figure 6: Geometrical interpretation of (R1). 


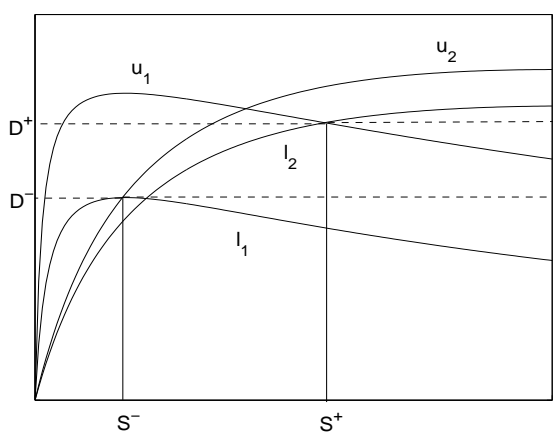

Figure 7: Location of points $D^{-}, D^{+}, s^{-}$and $s^{+}$.

Let us denote by $s^{-}$and $s^{+}$the points in $\left(0, s_{i n}\right)$ such that $s^{-}<s^{+}$and

$$
\begin{aligned}
& l_{1}\left(s^{-}\right)=u_{2}\left(s^{-}\right)=D^{-}>0, \\
& u_{1}\left(s^{+}\right)=l_{2}\left(s^{+}\right)=D^{+}>0 .
\end{aligned}
$$

(R2) $u_{1}(s)<l_{2}(s)$ for all $s \in\left(s^{+}, s_{i n}\right)$.

(R3) We have that $D^{+}>D^{-}$or

$$
y_{\min }>\frac{g^{-1}\left(D^{-}\right)-g^{-1}\left(D^{+}\right)}{s^{+}-s^{-}} \text {if } D^{-}>D^{+} .
$$

Let us build the system (4)- substituting $f_{1}, f_{2}$ by $l_{1}, u_{2}$ in system (4). Analogously, we build the system (4) ${ }^{+}$substituting $f_{1}, f_{2}$ by $u_{1}, l_{2}$ in system (4).

Let us denote by $\left(5^{-}\right)$and $\left(5^{+}\right)$the Eq. (5) with $f_{1}$ replaced by $l_{1}$ and $u_{1}$ respectively. Analogously we denote by $\left(6^{-}\right)$and $\left(6^{+}\right)$the Eq. (6) with $f_{2}$ replaced by $l_{2}$ and $u_{2}$ respectively.

We will make the assumptions for systems $\left(4^{-}\right)$and $\left(4^{+}\right)$:

(H1*) The following inequalities hold:

$$
\begin{aligned}
& g\left(y_{1}\left[s_{i n}-s^{-}\right]\right)>D^{-}>g\left(y_{2}\left[s_{i n}-s^{-}\right]\right), \\
& g\left(y_{1}\left[s_{i n}-s^{+}\right]\right)>D^{+}>g\left(y_{2}\left[s_{i n}-s^{+}\right]\right) .
\end{aligned}
$$

$\left(\right.$ H2$\left.^{*}\right)$ Eqs. $\left(5^{-}\right)$and $\left(6^{+}\right)$have one positive solution $\lambda_{1}^{-}$and $\lambda_{2}^{+}$respectively. Eqs. $\left(5^{+}\right)$and $\left(6^{-}\right)$have one positive solution $\lambda_{1}^{+}$and $\lambda_{2}^{-}$respectively. Moreover if $y_{1}>y_{2}$, then $\lambda_{1}^{-}, \lambda_{1}^{+}$are in $\left(s^{*}, s_{i n}\right)$ and $\lambda_{2}^{-}, \lambda_{2}^{+}$are in $\left(0, s^{*}\right)$. 
(H3*) The following inequalities hold:

$$
\begin{cases}y_{\min } g^{\prime}\left(x_{1}+x_{2}\right)>-l_{1}^{\prime}\left(s_{i n}-\frac{x_{1}}{y_{1}}-\frac{x_{2}}{y_{2}}\right) & \text { for all }\left(x_{1}, x_{2}\right) \in \mathcal{O}, \\ y_{\min } g^{\prime}\left(x_{1}+x_{2}\right)>-u_{1}^{\prime}\left(s_{i n}-\frac{x_{1}}{y_{1}}-\frac{x_{2}}{y_{2}}\right) & \text { for all }\left(x_{1}, x_{2}\right) \in \mathcal{O}\end{cases}
$$

$\left(\mathbf{H} 4^{*}\right)$ The following inequalities hold:

$$
\begin{cases}y_{\min } g^{\prime}\left(x_{1}+x_{2}\right)>-u_{2}^{\prime}\left(s_{i n}-\frac{x_{1}}{y_{1}}-\frac{x_{2}}{y_{2}}\right) & \text { for all }\left(x_{1}, x_{2}\right) \in \mathcal{O} \\ y_{\min } g^{\prime}\left(x_{1}+x_{2}\right)>-l_{2}^{\prime}\left(s_{i n}-\frac{x_{1}}{y_{1}}-\frac{x_{2}}{y_{2}}\right) & \text { for all }\left(x_{1}, x_{2}\right) \in \mathcal{O}\end{cases}
$$

Theorem 1 implies that $\left(s^{-}, x_{1}^{-}, x_{2}^{-}\right)$and $\left(s^{+}, x_{1}^{+}, x_{2}^{+}\right)$are solutions globally asymptotically stable of systems $(4)^{-}$and $(4)^{+}$respectively. Moreover $x_{i}^{-}$and $x_{i}^{+}(i=1,2)$ are defined by:

$$
\begin{array}{ll}
x_{1}^{-}=\frac{y_{1}\left[y_{2}\left(s_{i n}-s^{-}\right)-g^{-1}\left(D^{-}\right)\right]}{y_{2}-y_{1}}, & x_{2}^{-}=\frac{y_{2}\left[g^{-1}\left(D^{-}\right)-y_{1}\left(s_{i n}-s^{-}\right)\right]}{y_{2}-y_{1}} \\
x_{1}^{+}=\frac{y_{1}\left[y_{2}\left(s_{i n}-s^{+}\right)-g^{-1}\left(D^{+}\right)\right]}{y_{2}-y_{1}}, & x_{2}^{+}=\frac{y_{2}\left[g^{-1}\left(D^{+}\right)-y_{1}\left(s_{i n}-s^{+}\right)\right]}{y_{2}-y_{1}} .
\end{array}
$$

By assumption (R2) we have that $x_{1}^{-}<x_{1}^{+}$and $x_{2}^{+}<x_{1}^{-}$.

Theorem 2 Let $y_{\max }=y_{1}$, if the functions $f_{1}$ and $f_{2}$ are unknown but verify assumptions (R1)-(R3) and the functions $g, u_{i}, l_{i}(i=1,2)$ satisfy assumptions $\left(\mathbf{H} 1^{*}\right)-\left(\mathbf{H} 4^{*}\right)$, then the solutions of system (4) verify:

$$
\begin{aligned}
& x_{1}^{-} \leq \liminf _{t \rightarrow+\infty} x_{1}(t) \leq \limsup _{t \rightarrow+\infty} x_{1}(t) \leq x_{1}^{+}, \\
& x_{2}^{+} \leq \liminf _{t \rightarrow+\infty} x_{2}(t) \leq \limsup _{t \rightarrow+\infty} x_{2}(t) \leq x_{2}^{-}, \\
& s^{-} \leq \liminf _{t \rightarrow+\infty} s(t) \leq \limsup _{t \rightarrow+\infty} s(t) \leq s^{+} .
\end{aligned}
$$

In particular, we obtain that system (4) is uniformly persistent.

Proof: Note that, even if $f_{1}$ and $f_{2}$ are unknown, asymptotic behavior stated by Lemma (1) is still valid. Then we can proceed as in the proof of Theorem 1 and we need study only the $\omega$-limit set of the planar system (15). Moreover, we consider the restricted competitive system associate to $(4)^{-}$:

$$
\left\{\begin{array}{l}
\dot{v}_{1}=v_{1}\left(l_{1}\left(s_{i n}-\frac{v_{1}}{y_{1}}-\frac{v_{2}}{y_{2}}\right)-g\left(v_{1}+v_{2}\right)\right), \\
\dot{v}_{2}=v_{2}\left(u_{2}\left(s_{i n}-\frac{v_{1}}{y_{1}}-\frac{v_{2}}{y_{2}}\right)-g\left(v_{1}+v_{2}\right)\right) .
\end{array}\right.
$$

and the restricted competitive system associate to $(4)^{+}$:

$$
\left\{\begin{array}{l}
\dot{w}_{1}=w_{1}\left(u_{1}\left(s_{i n}-\frac{w_{1}}{y_{1}}-\frac{w_{2}}{y_{2}}\right)-g\left(w_{1}+w_{2}\right)\right), \\
\dot{w}_{2}=w_{2}\left(l_{2}\left(s_{i n}-\frac{w_{1}}{y_{1}}-\frac{w_{2}}{y_{2}}\right)-g\left(w_{1}+w_{2}\right)\right) .
\end{array}\right.
$$


Replacing (4) by (4)- and (4) ${ }^{+}$in Theorem 1 we obtain that:

$$
\begin{aligned}
& \lim _{t \rightarrow+\infty} v_{1}(t)=x_{1}^{-} \quad \text { and } \quad \lim _{t \rightarrow+\infty} v_{2}(t)=x_{2}^{-}, \\
& \lim _{t \rightarrow+\infty} w_{1}(t)=x_{1}^{+} \quad \text { and } \quad \lim _{t \rightarrow+\infty} w_{2}(t)=x_{2}^{+} .
\end{aligned}
$$

Let $\left(z_{1}, z_{2}\right)$ a solution of system (15) such that $z_{i}(0)=v_{i}(0)=w_{i}(0)$, Proposition 3 (see Appendix) implies that

$$
v_{1}(t) \leq z_{1}(t) \leq w_{1}(t) \quad \text { and } \quad w_{2}(t) \leq z_{2}(t) \leq v_{2}(t) \text { for all } t \geq 0 .
$$

Letting $t \rightarrow+\infty$, Proposition 2 implies (26) and the proof is complete.

Remark 4 Let $s_{1 l}^{*}$ and $s_{1 u}^{*}\left(s_{2 l}^{*}\right.$ and $\left.s_{2 u}^{*}\right)$ be the maximum of $l_{1}$ and $u_{1}\left(l_{2}\right.$ and $\left.u_{2}\right)$ respectively. In some cases, the relative order of those points allows us to drop some statements of assumptions $\left(\mathbf{H 3}^{*}\right)-\left(\mathbf{H} 4^{*}\right)$ :

If $s^{+}<\min \left\{s_{2 l}^{*}, s_{2 u}^{*}\right\}$, we can replace the function $u_{2}$ by an envelope $m_{2}$ as in the proof of case (ii) of Theorem 1, hence the first inequality in (25) is unnecessary and the proof of Theorem 2 runs as before.

If $s^{+}<\min \left\{s_{1 l}^{*}, s_{1 u}^{*}, s_{2 l}^{*}, s_{2 u}^{*}\right\}$ we can replace the functions $u_{i}$ by envelopes $m_{i}$ as in the proof of case (iii) of Theorem 1. Moreover, if we can build an envelope $m_{1}$ that does not intersects $l_{2}$ before $s_{i n}$, hence the second inequality in (24) and first inequality in (25) are unnecessary and the proof of Theorem 2 runs as before.

\section{$7 \quad$ Numerical example}

The system (4) is solved numerically using a couple of Haldane uptake functions:

$$
f_{1}(s)=\frac{2 s}{2+s+s^{2}} \quad \text { and } \quad f_{2}(s)=\frac{3 s}{6+s+s^{2}} .
$$

Hence, it follows that $s_{1}^{*}=\sqrt{2}, s_{2}^{*}=\sqrt{6}$ and $s^{*}=2$. The parameters of system were fixed at $s_{i n}=8, y_{1}=3$ and $y_{2}=1$. Moreover, we consider the family of feedback control laws:

$$
g_{\alpha}\left(x_{1}+x_{2}\right)=\frac{\alpha\left(x_{1}+x_{2}\right)}{5+\left(x_{1}+x_{2}\right)}, \quad \alpha>0 .
$$
to:

Assumption (H1) is verified for $\alpha \in I=(0.6389,0.9167)$ and Eqs. (5)-(6) are equivalent

$$
\begin{gathered}
3 \alpha s^{3}-(21 \alpha+6) s^{2}+(58-18 \alpha) s-48 \alpha=0, \\
\alpha s^{3}-(7 \alpha+3) s^{2}+(13-2 \alpha) s-48 \alpha=0 .
\end{gathered}
$$

Clearly, each polynomial has a positive real root $\lambda_{1}(\alpha)$ and $\lambda_{2}(\alpha)$ respectively and (H2) is verified in a subset of $I$. 



Figure 8: $\alpha=0.86$ and $\alpha=0.84$, case (i) of Theorem (1) is verified.
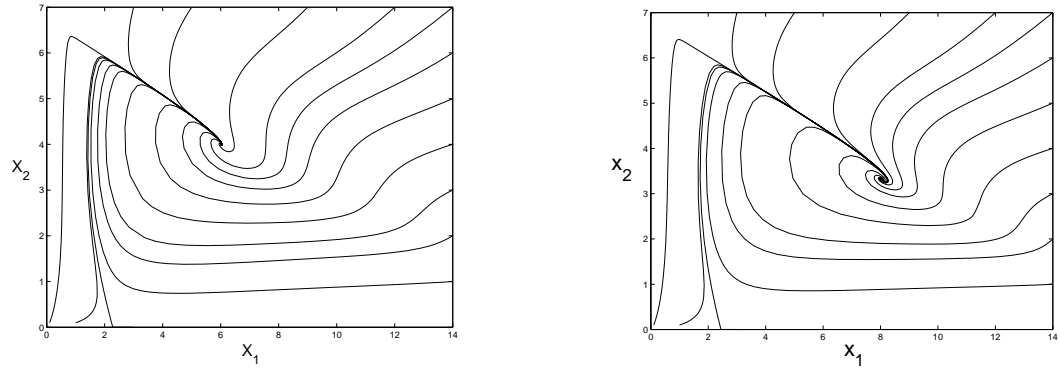

Figure 9: $\alpha=0.75$ and $\alpha=0.72$, case (ii) of Theorem (1) is verified.
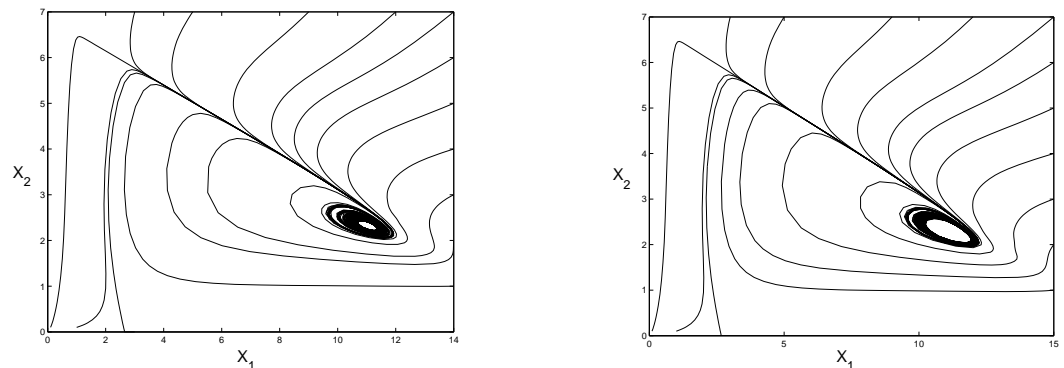

Figure 10: $\alpha=0.687$ and $\alpha=0.685$, the Theorem (1) is not verified but coexistence is achieved

Numerical simulations were carried out in MATLAB using ODE45 and they are presented in the Figs. 8, 9 and 10. We discuss briefly these results.

$\mathrm{RR} \mathrm{n}^{\circ} 5033$ 
For parameters $\alpha=0.86$ and $\alpha=0.84$, we can see with the help of computer that assumptions (H3)-(H4) are verified and Theorem 1 can be applied and Fig. 8 shows that $\left(s^{*}, x_{1}^{*}, x_{2}^{*}\right)$ is globally asymptotically stable.

For parameters $\alpha=0.75$ and $\alpha=0.72$, we can see that assumption (H3) is verified but not (H4). Owing to $s^{*} \in\left(s_{1}^{*}, s_{2}^{*}\right)$ it follows that the case (ii) of Theorem 1 can be applied and Fig. 9 shows that $\left(s^{*}, x_{1}^{*}, x_{2}^{*}\right)$ is globally asymptotically stable.

For parameters $\alpha=0.687$ and $\alpha=0.685$, we can see that assumptions (H3)-(H4) are not verified and Theorem 1 cannot be applied and Fig. 10 shows that $\left(s^{*}, x_{1}^{*}, x_{2}^{*}\right)$ is not stable but there is a limit cycle and the uniform persistence is achieved.

In the Fig. 10 we see that using $\alpha$ as a bifurcation parameter, for a critical value of $\alpha$ (determined by assumptions $(\mathbf{H} 1)-(\mathbf{H} 4))$, the positive equilibrium loses its stability and -under some conditions- a family of periodic solutions bifurcates from the positive equilibrium. Although the stability conditions of the bifurcation periodic solutions were not analytically given, numerical simulation was done to show that with suitable parameters, a stable limit cycle exists when the Hopf bifurcation parameter is near its critical value.

In all these cases, the uniform persistence of species has been obtained as a critical point globally asymptotically stable or an attractive periodic orbit.

\section{Discussion}

We have analyzed a model of the chemostat with competition such that the only output available is the total biomass. The main result is that, considering the dilution rate $D$ as a feedback control, one has - under some hypotheses- the uniform persistence of competing species in contrast to competitive exclusion in the classical chemostat. The novelty of this work is to consider nonmonotone uptake functions, generalizing in certain sense the result presented in [7].

The model took the form of a system of differential equations such that its asymptotic behavior is equivalent to a competitive planar differential system. Theory of asymptotically autonomous dynamical systems and theory of competitive dynamical systems played a prominent role.

If we consider $\varepsilon_{i}$ to be the specific death rate of species $x_{i}$ and we substitute $D$ by $D_{i}=D+\varepsilon_{i}$ in the equation (1), the tools mentioned above cannot be used. Handling different death rates remain an open question, worth of further study.

Moreover, from an experimental point of view, it would be very interesting to study the same problem considering $s_{i n}$ as the feedback control variable and the substrate $s$ as the output available.

One of the most strong assumptions in our model is $y_{\max }=y_{1}$. It is clear that we must consider other feedback control laws for the cases $y_{\max }=y_{2}$ and $y_{1}=y_{2}$. 


\section{Appendix}

In this section we state a result of comparison for competitive dynamical systems that is essential in the proof of Theorems 1 and 2 (see [14],[15] for more details).

Let the convex cone $K_{(0,1)}$ defined as

$$
K_{(0,1)}=\left\{\left(u_{1}, u_{2}\right) \in \mathbb{R}^{2}: u_{1} \geq 0 \text { and } u_{2} \leq 0\right\}
$$

and define a partial order in $\mathbb{R}^{2}$ by $\vec{y} \leq_{K_{(0,1)}} \vec{x}$ if $\vec{x}-\vec{y} \in K_{(0,1)}$, that means $y_{1} \leq x_{1}$ and $y_{2} \geq x_{2}$.

Let a continuous function $F: \Omega \mapsto \mathbb{R}^{2}$ where $\Omega$ is an open set in $\mathbb{R}^{2} . F=\left(F_{1}, F_{2}\right)$ is said to be of type $K_{(0,1)}$ if for each $i,(-1)^{m_{i}} F_{i}(\vec{a})<(-1)^{m_{i}} F_{i}(\vec{b})$ for any two points $\vec{a}$ and $\vec{b}$ in $\Omega$ satisfying $\vec{a} \leq_{K_{(0,1)}} \vec{b},\left(m_{1}, m_{2}\right)=(0,1)$ and $a_{i}=b_{i}$.

The object is to compare solutions of the system of differential equations:

$$
x^{\prime}=F(x) .
$$

with solutions of the systems of differential equations

$$
\begin{aligned}
& z^{\prime}=G(z) . \\
& y^{\prime}=H(y) .
\end{aligned}
$$

Such that the continuous functions $G, H: \Omega \mapsto \mathbb{R}^{2}$ verify the inequalities $F \leq_{K_{(0,1)}} G$ and $H \leq_{K_{(0,1)}} F$.

Proposition 3 (Comparison Theorem) Let $F$ be continuous on $\Omega$ and of type $K_{(0,1)}$. Let $x(t)$ be a solution of (29) defined on $[a, b]$. If $z(t)$ is a continuous function on $[a, b]$ satisfying (30) on $(a, b)$ with $z(a) \leq_{K_{(0,1)}} x(a)$, then $z(t) \leq_{K_{(0,1)}} x(t)$ for all $t$ in $[a, b]$. If $y(t)$ is a continuous function on $[a, b]$ satisfying $(31)$ on $(a, b)$ with $y(a) \geq_{K_{(0,1)}} x(a)$, then $y(t) \geq_{K_{(0,1)}} x(t)$ for all $t$ in $[a, b]$.

Proof: See lemma 2 from [14].

Note that, if system (29) is competitive and $\Omega$ is convex, then $F$ is of type $K_{(0,1)}$.

\section{References}

[1] P. Agrawal, G. Koshy and M. Ramseier. An algorithm for operating a fed-batch reactor at optimum specific rate. Biotechnol.Bioeng., 33:115-125, 1989.

[2] R.A. Armstrong and R. McGehee. Competitive exclusion. Amer.Natur., 115,2:151-170, 1980.

[3] J.F. Andrews. A mathematical model for the continuous culture of microorganisms utilizing inhibitory substrate. Biotechnol.Bioeng., 10:707-723, 1968. 
[4] G. Bastin and D. Dochain. On-line estimation and adaptative control of bioreactors, Elsevier, 1990.

[5] G.J. Butler and G.S.K. Wolkowicz. A mathematical model of the chemostat with a general class of functions describing nutrient uptake. SIAM J.Appl.Math., 45:138-151, 1985.

[6] G.J. Butler, H.I. Freedman and P. Waltman. Uniformly persistent systems. Proc.Am.Math.Soc., 96:425-430, 1986.

[7] P. De Leenheer and H. Smith. Feedback control for chemostat models. J.Math.Biol., 46:48-70, 2003.

[8] J. Hofbauer. A unified approach to persistence. Acta Appl.Math., 14:11-22, 1989.

[9] H.K.Khalil. Nonlinear Systems, Prentice Hall, 2002.

[10] L. Mailleret, O. Bernard and J.P. Steyer. Contrôle asymptotique non linéaire des fermenteurs anaérobies. Journal Européen des systèmes automatisés, 37,2:275-286, 2003.

[11] W. Sokol and J.A. Howell. Kinetics of phenol oxidation by washed cells. Biotechnol.Bioeng., 23:2039-2049, 1981.

[12] E.D. Sontag. Mathematical Control Theory, Texts in applied Mathematics, 6, SpringerVerlag, 1990.

[13] H. Smith and P. Waltman. The theory of the chemostat. Dynamics of microbial competition, Cambridge studies in mathematical biology, 13, Cambridge: Cambridge University Press, 1995.

[14] H. Smith. Dynamic of competition, in Mathematics inspired by biology, Springer Lecture Notes in Math, 1714:191-240, 1999.

[15] H. Smith. Monotone Dynamical Systems: an introduction to the theory of competitive and cooperative systems, Mathematical surveys and monographs, 41, Providence,RI,AMS, 1995.

[16] H. Thieme. Convergence results and a Poincaré-Bendixson trichotomy for asymptotically autonomous differential equations. J.Math.Biol., 30:755-763, 1992. 


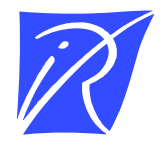

\section{Unité de recherche INRIA Sophia Antipolis 2004, route des Lucioles - BP 93 - 06902 Sophia Antipolis Cedex (France)}

Unité de recherche INRIA Futurs : Parc Club Orsay Université - ZAC des Vignes 4, rue Jacques Monod - 91893 ORSAY Cedex (France)

Unité de recherche INRIA Lorraine : LORIA, Technopôle de Nancy-Brabois - Campus scientifique 615, rue du Jardin Botanique - BP 101 - 54602 Villers-lès-Nancy Cedex (France)

Unité de recherche INRIA Rennes : IRISA, Campus universitaire de Beaulieu - 35042 Rennes Cedex (France)

Unité de recherche INRIA Rhône-Alpes : 655, avenue de l'Europe - 38334 Montbonnot Saint-Ismier (France)

Unité de recherche INRIA Rocquencourt : Domaine de Voluceau - Rocquencourt - BP 105 - 78153 Le Chesnay Cedex (France)

INRIA - Domaine de Voluceau - Rocquencourt, BP 105 - 78153 Le Chesnay Cedex (France)

http://www.inria.fr

ISSN 0249-6399 